\title{
Politiques d'éducation prioritaire en Catalogne : trajectoires et défaillances politiques
}

Xavier Bonal et Marcel Pagès

Traducteur : Sylvaine Herold

(2) OpenEdition

Journals

Édition électronique

URL : https://journals.openedition.org/ries/8922

DOI : $10.4000 /$ ries.8922

ISSN : 2261-4265

Éditeur

France Education international

Édition imprimée

Date de publication : 1 décembre 2019

Pagination : 22-25

ISBN : 9782854206258

ISSN : $1254-4590$

Référence électronique

Xavier Bonal et Marcel Pagès, « Politiques d'éducation prioritaire en Catalogne : trajectoires et défaillances politiques », Revue internationale d'éducation de Sèvres [En ligne], 82 | décembre 2019, mis en ligne le 01 décembre 2021, consulté le 15 janvier 2022. URL : http://journals.openedition.org/ries/ 8922 ; DOI : https://doi.org/10.4000/ries.8922

Ce document a été généré automatiquement le 15 janvier 2022.

(c) Tous droits réservés 


\title{
Politiques d'éducation prioritaire en Catalogne : trajectoires et défaillances politiques
}

\author{
Xavier Bonal et Marcel Pagès \\ Traduction : Sylvaine Herold
}

1 La trajectoire et la configuration des politiques d'éducation prioritaire en Catalogne doivent être observées dans leur contexte socio-historique spécifique et au regard de la tradition politique et administrative espagnole. En premier lieu, il convient de souligner l'importante inhibition de l'État dans le champ éducatif jusqu'au milieu des années 1980, lorsque la Loi sur le droit à l'éducation (LODE) a été approuvée (1985). Ce retard dans l'élaboration du modèle actuel de politique éducative s'explique principalement par le processus tardif de démocratisation en Espagne, mais est également lié à des facteurs politiques et partisans. L'inhibition de l'État en matière éducative a facilité le développement du secteur privé, principalement catholique, et l'élaboration d'un système d'enseignement mixte composé d'établissements privés financés par l'État et d'établissements publics. Les établissements publics sont devenus l'option scolaire des pauvres et, aujourd'hui encore, ils accueillent la majorité des élèves vulnérables. Ce système éducatif dual a été consolidé par un ensemble de réglementations de quasi-marché, qui ont renforcé les politiques de choix scolaire et affaibli les mécanismes potentiels de réduction des inégalités. De ce fait, les systèmes éducatifs espagnol et catalan connaissent des niveaux importants de ségrégation scolaire. De plus, dans le cas de la Catalogne, ce processus a eu lieu dans un contexte politique marqué par la prééminence évidente d'agendas politiques néoconservateurs, opposés à la prise en compte systématique et coordonnée des établissements et territoires socialement défavorisés.

2 Ce processus historique explique le retard de développement des politiques d'éducation prioritaire en Catalogne. Ce n'est qu'au début des années 1980 que le gouvernement catalan a commencé à élaborer des mesures visant à soutenir les établissements socialement défavorisés. On peut identifier trois grandes phases dans l'élaboration des 
politiques de compensation. La première phase commence avec l'adoption du Programme d'éducation compensatoire (PEC) en 1983, qui a mis au point une approche palliative et sélective s'adressant à des groupes cibles spécifiques, en particulier les minorités ethniques, telles que les enfants roms dans des quartiers spécifiques. Le PEC a doté les établissements de fonds (limités) et de ressources humaines supplémentaires, afin de faciliter l'intégration scolaire des élèves vulnérables. Des repas gratuits ou des financements pour acheter le matériel scolaire ont également été distribués aux familles bénéficiaires les plus pauvres. L'ontologie politique sous-tendant le PEC se fonde sur la théorie du déficit culturel et part de l'idée qu'une augmentation des ressources destinées aux établissements ayant le plus de difficultés favorisera la scolarisation des élèves vulnérables en " compens[ant] les difficultés particulières » qui découlent de leurs « conditions sociales et culturelles".

3 La deuxième phase n'a eu lieu que début 2000, dans le contexte de l'apparition de nouveaux besoins sociaux et éducatifs dans les établissements, du fait de la croissance de flux migratoires émergents. L'intensification de l'arrivée d'élèves migrants (en une décennie, les élèves étrangers sont passés de $2 \%$ à $15 \%$ de la population scolaire totale) a obligé le gouvernement catalan à formuler des réponses politiques immédiates, afin de faciliter leur inclusion. Cette deuxième période se caractérise par un modèle basé sur l'intégration linguistique, l'éducation interculturelle et la cohésion sociale. La mesure paradigmatique de cette phase est le Plan de cohésion linguistique et sociale, approuvé en 2004, qui contenait des mesures visant à promouvoir l'intégration des élèves migrants au sein des établissements scolaires et de la société, et plusieurs stratégies d'appui pédagogique et linguistique. L'approche en termes de déficit culturel a été mise de côté et un nouveau paradigme, fondé sur le droit à l'éducation et l'attention portée à la diversité, a été adopté comme cadre principal de l'intervention politique dans les établissements défavorisés.

4 La dernière période débute avec l'approbation de la première loi catalane sur l'éducation, adoptée en 2009 par le Parlement catalan. Cette loi met en œuvre l'autonomie des établissements, la professionnalisation du leadership et une nouvelle architecture de la gouvernance éducative comme principales stratégies de réforme du système éducatif. Quelques années après l'approbation de cette réforme, un nouveau modèle d'identification et de classification des établissements a émergé. Les établissements sont désormais classés selon leur « complexité sociale ». Le département de l'éducation catalan identifie et classe les établissements en trois groupes selon des indicateurs de différente nature, tels que des facteurs socioéconomiques (familles exerçant des emplois peu qualifiés, familles sans emploi et parents bénéficiaires du revenu minimum), des indicateurs éducatifs (pourcentage d'élèves ayant des besoins spéciaux) et des indicateurs socioculturels (pourcentage d'élèves migrants). Selon cette méthodologie, $17 \%$ de l'ensemble des établissements catalans d'enseignement primaire et $16 \%$ des établissements d'enseignement secondaire sont considérés d'une complexité maximale, la plupart appartenant au secteur public (Bonal et Pagès, 2019). La liste des établissements classés comme relevant d'une complexité sociale maximale est publiée par décret par le département de l'éducation.

5 Pour ces établissements, le département de l'éducation catalan prévoit une plus grande autonomie pour la sélection et l'affectation des enseignants, en dehors du système centralisé. Ils sont également susceptibles de recevoir davantage de ressources humaines et matérielles que les établissements standards. Ce nouveau système de 
classification et de publication des établissements a suscité de vives préoccupations quant à la stigmatisation potentielle de ces établissements du fait de leur composition sociale. En outre, les avantages liés à une meilleure identification des besoins scolaires ne se sont pas traduits par des politiques éducatives plus transparentes et mieux coordonnées. De fait, aucune réglementation publique officielle n'établit les ressources compensatoires liées aux différents niveaux de complexité sociale des établissements. Des marges de manœuvre importantes existent donc pour des pratiques discrétionnaires en matière d'affectation des ressources. En outre, aucun système d'incitations n'est prévu pour encourager les enseignants à travailler dans ces établissements. La formation des enseignants pour exercer dans des établissements socialement complexes est totalement absente, et le département de l'éducation n'a défini aucune $=$ politique en matière de carrière professionnelle des enseignants pour travailler dans ces établissements, contrairement aux politiques développées dans des pays comme le Royaume-Uni ou la France (Karsten, 2006). En résumé, la tentative d'améliorer l'identification et la classification de la complexité sociale des établissements contraste avec l'absence de stratégie politique visible déterminant les ressources dont ces établissements ont besoin et le type d'interventions requises pour améliorer leurs performances scolaires et l'inclusion sociale.

6 Les effets de ces politiques ne sont pas particulièrement encourageants. Les politiques d'éducation prioritaire sont demeurées rares en Catalogne et n'ont eu que peu d'impact sur l'amélioration de la situation des établissements et des élèves les plus défavorisés. Les principaux indicateurs éducatifs stagnent et montrent que la situation sociale des établissements et des élèves continue de conditionner les inégalités en termes d'accès à l'éducation, de conditions de scolarisation et de résultats scolaires. Les résultats scolaires aux tests nationaux sont nettement moins bons dans les établissements socialement complexes (Bonal et Pages, 2019). De plus, on constate un effet white flight ${ }^{1}$ évident dans ces établissements: les familles des classes moyenne et moyenne inférieure perçoivent un risque à inscrire leurs enfants dans des établissements à forte concentration d'élèves aux besoins d'apprentissage élevés. Par conséquent, le niveau de ségrégation scolaire des élèves issus de milieu socioéconomique défavorisé ou de l'immigration demeure élevé et est resté presque constant au cours de la décennie passée (Síndic de Greuges, 2016).

7 En résumé, une brève analyse de la trajectoire politique des politiques d'éducation prioritaire en Catalogne suggère des lacunes importantes. Premièrement, il y a un manque évident de stratégie politique coordonnée et systématique pour répondre aux besoins des établissements et des élèves les plus vulnérables. Deuxièmement, l'orientation de ces politiques se caractérise par des omissions politiques importantes, telles que l'absence d'une approche territoriale de l'intervention éducative ou d'une plus grande attention portée aux mesures préventives plutôt que palliatives. Troisièmement, les politiques d'éducation prioritaire n'ont pas mis en œuvre de stratégies spécifiques en matière de formation des enseignants ni d'incitations professionnelles pour encourager les enseignants spécialisés à travailler dans ces établissements. Enfin, les politiques existantes visant à réduire l'échec scolaire ou le décrochage scolaire précoce n'ont eu qu'un impact mineur. Dans l'ensemble, nous estimons qu'un nouveau programme politique en faveur de la scolarisation dans les établissements et territoires les plus vulnérables est nécessaire. Il devra mettre au point des approches novatrices pour répondre aux principaux défis politiques soulevés dans le domaine de l'éducation prioritaire. Une question centrale est de savoir 
comment développer une politique d'éducation prioritaire sans une approche implicite en termes de déficit culturel et social. À cet égard, nous défendons l'idée que des approches plus universalistes des politiques éducatives devraient être privilégiées et qu'il faudrait passer de mesures palliatives à des mesures émancipatrices. En particulier, une intervention préventive basée sur les territoires et transcendant les politiques éducatives pourrait être un point de départ intéressant pour explorer de nouveaux modèles d'intervention politique afin de surmonter les limites du modèle actuel. De plus, le département de l'éducation doit définir un nouveau modèle de carrière professionnelle pour des enseignants hautement préparés à travailler dans ces contextes sociaux complexes. Le principe de transférabilité pédagogique n'est pas opérationnel lorsqu'on travaille dans des établissements caractérisés par la concentration de problèmes sociaux - pauvreté, chômage, abandon familial et manque de capital social. C'est pourquoi une formation spécifique et un système d'incitations visant à garantir que les meilleurs enseignants sont affectés aux établissements les plus complexes socialement sont nécessaires. Enfin, comme le faisait remarquer Bernstein il y a quelques années, "l'éducation ne peut compenser la société ». Cela implique la nécessaire coordination de l'action des différents départements liés aux politiques sociales. L'intervention éducative doit s'articuler avec l'action des départements en charge de la santé, de la culture, des services sociaux ou de l'emploi. Si l'on entend améliorer les expériences éducative et d'apprentissage des élèves, les politiques publiques doivent prendre en compte ce qui se passe en dehors de l'école.

\section{BIBLIOGRAPHIE}

BONAL X. et PAGÈS M. (2019). « Les polítiques educatives d'atenció als centres socialment desafavorits: anàlisi i propostes ». In J. RIERA (dir.), Reptes de l'educació a Catalunya. Anuari 2018. Barcelone : Fundació Jaume Bofill, p. 395-445.

KARSTEN S. (2006). «Policies for disadvantaged children under scrutiny: the Dutch policy compared with policies in France, England, Flanders and the USA ». Comparative education, $\mathrm{n}^{\circ} 42$ (2), p. 261-282.

SÍNDIC DE GREUGES (2016). La segregació escolar a Catalunya (I): la gestió del procés d'admissió d'alumnat. Síndic de Greuges de Catalunya.

\section{NOTES}

1. Ou «fuite des blancs» en français, expression qui désigne le phénomène de migration des personnes d'origine européenne hors des zones où le taux d'immigration des populations non blanches augmente significativement. (NdT) 


\section{INDEX}

Mots-clés : politique éducative, éducation prioritaire, exclusion sociale

Palabras claves : política educacional, exclusión social, educación prioritaria

Keywords : educational policy, social exclusion, priority education

Index géographique : Espagne

\section{AUTEURS}

\section{XAVIER BONAL}

Xavier Bonal est professeur de sociologie à l'Université autonome de Barcelone (UAB) et enseigne également à l'Université d'Amsterdam (UvA) dans les champs de l'éducation et du développement international. Il dirige le groupe de recherche Globalisation, Education and Social Policies (GEPS) au sein de l'UAB et coordonne GLOBED Project, un Master du programme Erasmus Mundus intitulé Education Policies for Global Development. Il est membre du Réseau européen d'experts en sciences sociales et de l'éducation (NESSE) et du comité de rédaction de revues internationales spécialisées dans les politiques et le développement de l'éducation. Auteur de nombreux ouvrages sur la sociologie de l'éducation, les politiques éducatives et la mondialisation, l'éducation et le développement, il est également consultant auprès d'organisations internationales. De 2006 à 2010, il a été médiateur adjoint aux droits de l'enfant auprès du médiateur de Catalogne. Courriel : xavier.bonal@uab.cat

\section{MARCEL PAGÈS}

Marcel Pagès est doctorant au département de sociologie de l'Université autonome de Barcelone. Ses centres d'intérêt portent sur la sociologie politique de l'éducation, avec une attention particulière aux questions relatives au processus de réforme, à la gouvernance et aux inégalités dans le champ de l'éducation. Courriel : marcelpages.pm@gmail.com 